\title{
Multiscale Morphological Image Simplification
}

\author{
Leyza Baldo Dorini and Neucimar Jerônimo Leite* \\ Institute of Computing, P.O. Box: 6176, \\ University of Campinas - UNICAMP, \\ 13084-971, Campinas, SP, Brazil \\ \{ldorini, neucimar\}@ic.unicamp.br
}

\begin{abstract}
Image simplification reduces the information content of an image, being frequently used as a preprocessing stage in several algorithms to suppress undesired details such as noise. Morphological filters, commonly used for this purpose, have as main drawbacks the asymmetric treatment of peaks and valleys and the difficulty to choose an appropriate structuring element size. Here, we propose a self-dual multiscale image simplification operator with sound edge preservation properties. This enables us to represent the inherent multiscale nature of real-world images by embedding the original signal into a family of derived signals, which represent simplified versions of the image obtained by successively removing its structures across scales. Thus, it is possible to analyze the different representation levels to extract the interest features, and the definition of a structure element size does not constitute a problem anymore. Based on these notions, we present some experiments on image segmentation, a basic step of various pattern recognition approaches.
\end{abstract}

Keywords: mathematical morphology, multiscale analysis, image segmentation, image simplification.

\section{Introduction}

In a morphological framework, image segmentation is typically done by first extracting markers of significant structures, and then using the watershed transform [1] to extract the contours of these structures as accurately as possible. However, image extrema (frequently used as markers) can correspond to insignificant structures or noise, and the gradient image, often associated to the watershed algorithm, naturally yields high responses in textured areas, for example. Thus, to prevent over-segmentation, unacceptable for further pattern recognition tasks, image simplification is an essential pre-processing stage when dealing with this segmentation paradigm.

In the linear approach, simplified images can be generated by convolving the original image with a Gaussian kernel. Its drawbacks, concerning mainly contour blurring and shifting, have motivated the development of non-linear approaches, such as the morphological and median filters [2] 3].

\footnotetext{
* The authors are grateful to FAPESP (07/52015-0; 05/04462-2) and MCT/CNPq $(472402 / 2007-2)$.
} 
The simplest morphological filters, openings and closings, simplify an image by respectively suppressing peaks and eliminating valleys, but displace horizontal edges 4 5. On the other hand, filters by reconstruction can reconstruct whole objects preserving the exact location of their edges. These filters transform the image by suppressing some of its components and then reconstructing those structures not eliminated in the initial step [6].

In fact, these transformations belong to a broader class of filters called connected operators, which transform the image by removing and merging flat zones. These filters present a superior performance in many image processing applications because they do not compromise shape information as it is the case for the morphological opening (closing) and the well-known linear filters 3 .

One drawback of the reconstruction filters is that they are not self-dual, that is, they asymmetrically treat background and foreground. A symmetric treatment can be obtained by using alternating sequential filters, which have a high computational cost, or the levelings, a more general class of morphological filters introduced by Meyer [2. Area opening [7] and openings and closings by reconstruction are special cases of levelings. Another drawback of morphological filters is the choice of a suitable structuring element that presents a good trade-off between simplification of large image components and preservation of important perceptually sensitive details.

In this paper, we propose a general multiscale morphological simplification operator that is self-dual. Besides the symmetric treatment of peaks and valleys, the multiscale structure enables us to analyze the different representation levels and explore the specific features that become explicit at each scale. We also explore the iterative application of the operator, showing via experiments that it properly merges flat zones.

\section{Multiscale Morphology}

Let $f: \mathcal{D} \subset \mathbb{R}^{n} \rightarrow \mathbb{R}$ be an image function and $g: \mathcal{G} \subset \mathbb{R}^{n} \rightarrow \mathbb{R}$ be a structuring function. The two fundamental operations of gray-scale morphology, erosion and dilation, are defined in the scaled form as:

Definition 1. [8] (Dilation) The dilation of the function $f(\boldsymbol{x})$ by the structuring function $g_{\sigma}(\boldsymbol{x}),\left(f \oplus g_{\sigma}\right)(\boldsymbol{x})$, is given by:

$$
\left(f \oplus g_{\sigma}\right)(\boldsymbol{x})=\sup _{t \in \mathcal{G} \cap \check{\mathcal{D}}_{-x}}\{f(\boldsymbol{x}-\boldsymbol{t})+g(\boldsymbol{t})\} .
$$

Definition 2. [8] (Erosion) The erosion of the function $f(\boldsymbol{x})$ by the structuring function $g_{\sigma}(\boldsymbol{x}),\left(f \ominus g_{\sigma}\right)(\boldsymbol{x})$, is given by:

$$
\left(f \ominus g_{\sigma}\right)(\boldsymbol{x})=\inf _{t \in \mathcal{G} \cap \mathcal{D}_{-x}}\{f(\boldsymbol{x}-\boldsymbol{t})-g(\boldsymbol{t})\} .
$$

where where $\mathcal{D}_{x}$ is the translate of $\mathcal{D}, \mathcal{D}_{x}=\{\mathbf{x}+\mathbf{t}: \mathbf{t} \in \mathcal{D}\}$, and $\check{\mathcal{D}}$ is the reflection of $\mathcal{D}$, which denotes the domain of the structuring function. 
The notion of scale comes from the use of the scaled structuring function $g_{\sigma}: \mathcal{G}_{\sigma} \subset \mathbb{R}^{2} \rightarrow \mathbb{R}$, such that 8 ]

$$
g_{\sigma}(\mathbf{x})=|\sigma| g\left(|\sigma|^{-1} \mathbf{x}\right) \quad \mathbf{x} \in \mathcal{G}_{\sigma}, \forall \sigma \neq 0,
$$

where $\mathcal{G}_{\sigma}=\{\mathbf{x}:\|\mathbf{x}\|<\mathcal{R}\}$ is the support region of the function $g_{\sigma}$ and $\sigma$ represents the scale.

To ensure reasonable scaling behavior, some conditions are necessary [8], requiring a monotonic decreasing structuring function along any radial direction from the origin. To avoid level-shifting and horizontal translation effects, respectively, one must also consider that $\sup _{\mathbf{t} \in \mathcal{G}_{\sigma}}\left\{g_{\sigma}(\mathbf{t})\right\}=0$ and $g_{\sigma}(\mathbf{0})=0$ [8].

Here, we carried out tests using the pyramid structuring function, given by $g(x, y)=-\max \{|x|,|y|\}$, that in the scaled version is

$$
g_{\sigma}(x, y)=-\sigma^{-1} \max \{|x|,|y|\} .
$$

Observe that, for a $3 \times 3$ structuring element (used in this work), $g_{\sigma}$ is zero at position $\mathbf{0}$ and $-\sigma^{-1}$ otherwise. We have dropped the || symbol on Equation 4 to emphasize that we only work with positive scales.

\section{The Operator Definition}

A toggle operator has two major points: the primitives and a given decision rule 6. Here, we use as primitives an extensive and an anti-extensive transformation. The decision rule involves, at a point $\mathbf{x}$, the value $f(\mathbf{x})$ and the primitives' results. Formally:

$$
\left(f \oslash g_{\sigma}\right)^{k}(\mathbf{x})=\left\{\begin{array}{l}
\psi_{1}^{k}(\mathbf{x}) \text { if } \psi_{1}^{k}(\mathbf{x})-f(\mathbf{x})<f(\mathbf{x})-\psi_{2}^{k}(\mathbf{x}), \\
f(\mathbf{x}) \text { if } \psi_{1}^{k}(\mathbf{x})-f(\mathbf{x})=f(\mathbf{x})-\psi_{2}^{k}(\mathbf{x}), \\
\psi_{2}^{k}(\mathbf{x}) \text { otherwise, }
\end{array}\right.
$$

where $\psi_{1}^{k}$ is an extensive transformation and $\psi_{2}^{k}$ is an anti-extensive transformation, both applied iteratively $k$ times. In this paper, we have used as primitives the the scale dependent dilation and erosion, that is, $\psi_{1}^{k}(\mathbf{x})=\left(f \oplus g_{\sigma}\right)^{k}(\mathbf{x})$ and $\psi_{2}^{k}(\mathbf{x})=\left(f \ominus g_{\sigma}\right)^{k}(\mathbf{x})$. We recall that the use of other primitives may lead to different properties, like causality or filtering, for example.

Proposition 1 states that the above defined operator is self-dual when using dual transformations as primitives.

Proposition 1. Consider two dual primitives $\psi$ and $\phi$, that is, $\psi(\boldsymbol{x})=\phi\left(\boldsymbol{x}^{*}\right)^{*}$. If $\psi$ is extensive and $\phi$ is anti-extensive, the toggle operator given by

$$
\mathcal{T}(\boldsymbol{x})=\left\{\begin{array}{l}
\psi(\boldsymbol{x}) \text { if } \psi(\boldsymbol{x})-f(\boldsymbol{x})<f(\boldsymbol{x})-\phi(\boldsymbol{x}), \\
f(\boldsymbol{x}) \text { if } \psi(\boldsymbol{x})-f(\boldsymbol{x})=f(\boldsymbol{x})-\phi(\boldsymbol{x}), \\
\phi(\boldsymbol{x}) \text { otherwise }
\end{array}\right.
$$

is a self-dual operator. 
ProOF. Follows directly from the complement operator definition.

Now, we define the multiscale representation scheme used in this work.

Definition 3. [3] (Pyramid of operators) A pyramid of operators is a family of operators $\left\{\Psi_{\sigma}\right\}$ depending of a positive parameter $\sigma$ such that, for each $\sigma \geq$ $\mu \geq 0$, there exists a given $\nu \geq 0$ such that $\Psi_{\nu} \Psi_{\mu}=\Psi_{\sigma}$.

Let us consider $\sigma$ as a scale parameter. This definition states that an image at scale $\sigma$ can be obtained either directly (there is no need of make an observation at all the smaller scales) or incrementally (in such a way that the image observed at scale $\mu+\nu$ is independent of the initial scale $\mu$ ). This is called the atlas principle $[5]$. Also, according to the definition, the composition of two operators of the family is still an operator of the family. Note that the composition rule can vary.

In the following, we show that the scaled dilation and erosion satisfy a semigroup property when using convex structuring functions. Thus, they can be used to build a pyramid of operators.

Proposition 2. (Semigroup property of convex structuring functions) A family $g_{\sigma}(\sigma>0)$ of scaled structuring functions, which are convex, is a one-parameter continuous semigroup. That is, $g_{\sigma} \oplus g_{\mu}=g_{\sigma+\mu}$ for $\sigma, \mu \geq 0$.

Proof. See [8].

This property, together with the chain rule for the dilation and erosion 44:

$$
f \oplus\left(g_{\sigma} \oplus g_{\mu}\right)=\left(f \oplus g_{\sigma}\right) \oplus g_{\mu} \text { and } f \ominus\left(g_{\sigma} \oplus g_{\mu}\right)=\left(f \ominus g_{\sigma}\right) \ominus g_{\mu} .
$$

with $g_{\sigma}(\mathbf{x})$ defined as in Equation 3 , lead directly to the semi-group property for the scale parametrized morphological operations:

$$
\begin{aligned}
& f \oplus g_{\sigma+\mu}=\left(f \oplus g_{\sigma}\right) \oplus g_{\mu}, \\
& f \ominus g_{\sigma+\mu}=\left(f \ominus g_{\sigma}\right) \ominus g_{\mu} .
\end{aligned}
$$

We now analyze the variation on the number of iterations, $k$. Let $g_{\sigma}$ be the pyramid structuring function (Equation 4). In this case, the atlas principle does not apply, but we have the following equivalence for the defined operator:

$$
\left(f \oslash g_{\sigma_{3}}\right)^{k}(\mathbf{x})==\left(f \oslash g_{\sigma_{2 k+1}}\right)^{1}(\mathbf{x}),
$$

where the subscript on $\sigma$ indicates the structuring element size. In a few words, to perform $k$ iterations of the primitives using a $3 \times 3$ structuring element and then apply the toggle operator is equivalent to perform one iteration using a structuring element of size $2 k+1$. In this way, a wider neighborhood is being considered, and regions tend to be merged. The defined operator can be seen as a quasi-connected operator, in the sense that it simplifies the image by creating quasi-flat zones.

Definition 4. Adapted from [2] (Quasi-flat zone) Two pixels x,y belong to the same quasi-flat zone of a function $f$ if and only if there exists an $n$-tuple of pixels $\left(p_{1}, p_{2}, \ldots, p_{n}\right)$ such that $p 1=x$ and $p n=y$, and for all $i,\left(p_{i}, p_{i+1}\right)$ are neighbors and satisfy the symmetrical relation $\left|f_{p_{i}}-f_{p_{i+1}}\right| \leq \lambda$. 


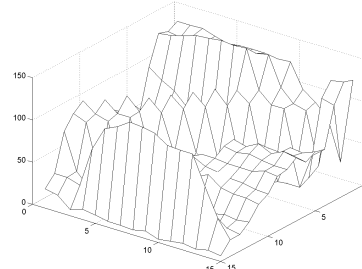

$(\mathrm{a})^{15}$

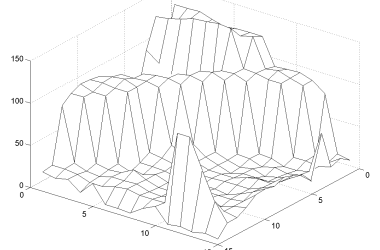

(b)

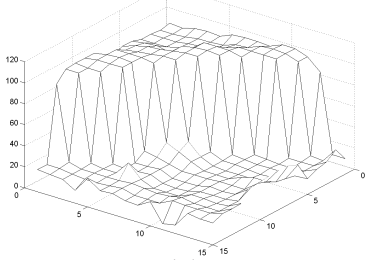

(c)

Fig. 1. Simplification obtained by considering successive iterations (1, 3 and 5$)$ of the operator at scale $\sigma=1$

Figure1 1illustrates the creation of these quasi-flat zones. We plot the gray levels of a small portion of an image when transformed by successive iterations of the defined operator, $k=1 \ldots 5$, with $\sigma=1$. Note that quasi-flat zones are created.

In Figure 2, we show the resulting images after applying the operator defined in Equation 5 for different scales and number of iterations. Note that the number of image structures decrease with the increase of scale.
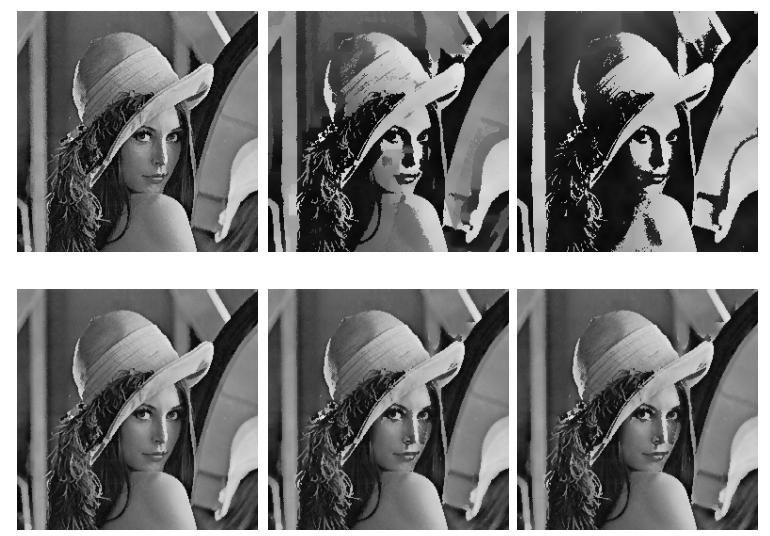

Fig. 2. The rows present the images processed by the defined operator at scales 1 and 0.1 , respectively. The columns represent 1,10 and 50 iterations.

In fact, it can be proved [9] that the defined operator has interesting scalespace properties when using the scaled dilation and erosion as primitives. Also observe that the increasing on the number of iterations yields a greater simplification. In the following, we use the defined image simplification operator as a preprocessing stage in different applications.

\section{Results}

In the first experiment, we use the h-maxima transform [6] to suppress all image maxima whose contrast is lower than a specified value $h$, and use the remaining 


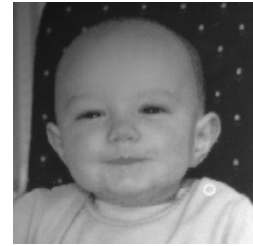

(a)

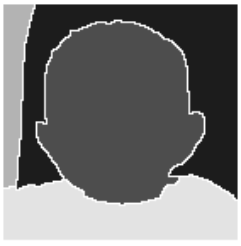

(b)

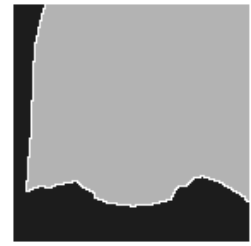

(c)

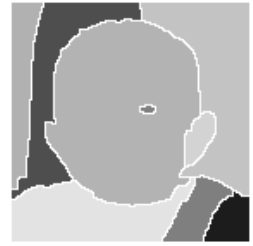

(d)

Fig. 3. Segmentation results for the facel image with non-uniform illumination [10]. (a) Original image, (b) using transformed image $\sigma=10$ and $k=5$, with $h=45$, (c) using an image transformed by an opening by reconstruction with $h=9$, and (d) based only on the original image using $h=45$.

extended extrema as markers in a watershed transform. Figure 3 illustrates the best results for each case. Observe that the image transformed by the defined operator (Figure 3(b)) is less sensitive to the illumination features. This example also shows that, in some cases, it is not enough to select image extrema according to some criteria, and a simplification step is essential.

The main difficulty that arises when using markers from an image processed by an opening by reconstruction is that the simplification may conduce to a merging of regions that disturbs the extraction of markers, as illustrated in Figure 4.

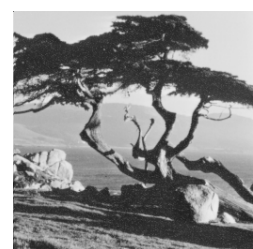

(a)

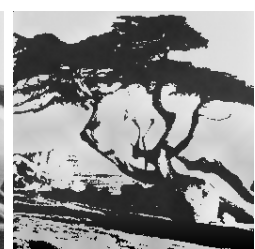

(b)

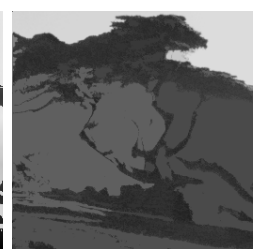

(c)

Fig. 4. (a) Original image and its simplification results based on (b) our operator, and (c) the opening by reconstruction

These images represent the maximum possible reduction on the number of flat zones (corresponding to 32 iterations for the defined operator and to a structuring element of size 63 for the opening by reconstruction). Although openings by reconstruction yields less flat zones, observe that the simplification for our operator is more suitable for marker extraction purposes.

When applying the defined operator, in some neighborhood of an important minimum (maximum), the pixels values will be eroded (dilated) in such a way that it is possible to identify the significant extrema of the image and their influence zones. In this sense, we define a new thresholding operation as follows:

$$
\left(f \oslash g_{\sigma}\right)^{k}(\mathbf{x})=\left\{\begin{array}{l}
255 \text { if } \psi_{1}^{k}(\mathbf{x})-f(\mathbf{x})<=f(\mathbf{x})-\psi_{2}^{k}(\mathbf{x}) \\
0 \quad \text { otherwise, }
\end{array}\right.
$$




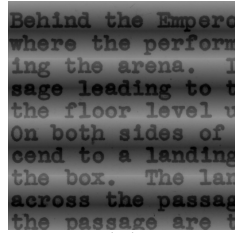

(a)

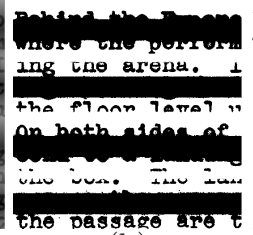

(b)

Behind the Empero DeuLuu wue smpor. Behind the Empero where the perform mann tha unmanm where the perform ing the arena. I Ing the arena. I sage leading to $t$ sage leading to $t$ sage leading to $t$ the floor level $u$ the floor level $u$ On both sides of vas uns cend to a landing nand tin a Tandino cend to a landing the box. The lan the box. The lan across the passag across the passag across the passag

Fig. 5. Binarization results. (a) original image, (b) Otsu, (c) Moving Averages, (d) Niblack and (e) Our approach.

and use as primitives the scaled erosion and dilation operations. Figure 5 shows the segmentation of a document image with non-uniform illumination. We compare our results against some well-known approaches, namely, the Otsu's, the moving averages' and the Niblack's algorithms [10.

The Otsu's algorithm, which defines a global threshold, was largely affected by the illumination conditions. The Niblack's algorithm wrongly classify all the pixels belonging to the darker areas of the image as background. Although our results seem comparable to that obtained by the moving averages' algorithm, the latter produces some stripes in the final result, as illustrated in Figure 6. This fact can disturb automatic optical character recognition (OCR) results.

\section{arena. arena.}

(a)

Fig. 6. (a) Our approach result and (b) The moving averages algorithm yields white stripes that may disturb OCR results

Figure 7 shows an example where the iterative application of the operator leads to a contour regularization. Note that the gradient extracted from the original image is weak, which can cause a leaking of the watershed function and, consequently, define a wrong segmentation result. Also, this scheme can be applied to noisy images, since the simplification performs a filtering on the original signal, while preserving and enhancing its boundaries.

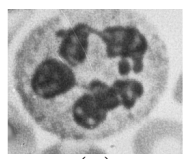

(a)

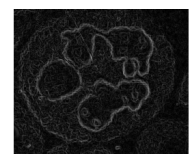

(b)

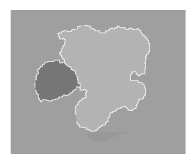

(c)

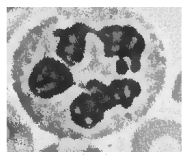

(d)

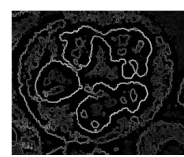

(e)

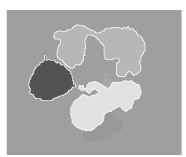

(f)

Fig. 7. Improvement of the gradient image by applying a scale-space toggle operator. (a-d) Original and regularized images, (b-e) the weak and the well-defined contours, (c-f) the different segmentation results. 


\section{Conclusions}

In this work, we have introduced a multiscale morphological simplification algorithm where we explore the variation on scale and number of iterations parameters to create simplified versions of an image. Experimental results show that the defined operator conduces to better segmentation and binarization results.

Also, the image contour regularization defined by the proposed operator has a better quality than the results obtained by traditional methods based on the high-boost filtering and the Laplacian operator. Further works on this matter include the analysis of other primitives and their properties aiming at improving and extending our initial results on image filtering, segmentation and simplification.

\section{References}

1. Beucher, S., Meyer, F.: The morphological approach to segmentation: the watershed transformation. In: Mathematical Morphology in Image Processing, pp. 433451. Marcel Dekker, New York (1993)

2. Meyer, F.: Levelings, image simplification filters for segmentation. Journal of Mathematical Imaging and Vision 20, 59-72 (2004)

3. Salembier, P., Serra, J.: Flat zones filtering, connected operators, and filters by reconstruction. IEEE Transactions on Image Processing, 1153-1160 (1995)

4. Serra, J.: Image Analysis and Mathematical Morphology. Theoretical Advances, vol. 2. Academic Press, London (1988)

5. Heijmans, H., van den Boomgaard, R.: Algebraic framework for linear and morphological scale-spaces. Journal of Visual Communication and Image Representation 13, 269-301 (2002)

6. Soille, P.: Morphological Image Analysis: Principles and Applications. Springer, Heidelberg (2003)

7. Vincent, L.: Grayscale area openings and closings: their applications and efficient implementation. In: EURASIP Workshop on Mathematical Morphology and its Applications to Signal Processing, pp. 22-27 (1993)

8. Jackway, P.T., Deriche, M.: Scale-space properties of the multiscale morphological dilation-erosion. PAMI 18, 38-51 (1996)

9. Dorini, L.E.B., Leite, N.J.: A scale-space toggle operator for morphological segmentation. In: 8th ISMM, pp. 101-112 (2007)

10. Parker, J.R.: Algorithms for Image Processing and Computer Vision. Wiley, Chichester (1996) 